POS PROCEEDINGS

\title{
Frank N. Bash Symposium 2011: New Horizons in Astronomy
}

Edited by Sarah Salviander, Joel Green, and Andreas Pawlik University of Texas at Austin

Frank N. Bash Symposium: New Horizons In Astronomy, October 9-11, 2011

Austin Texas 


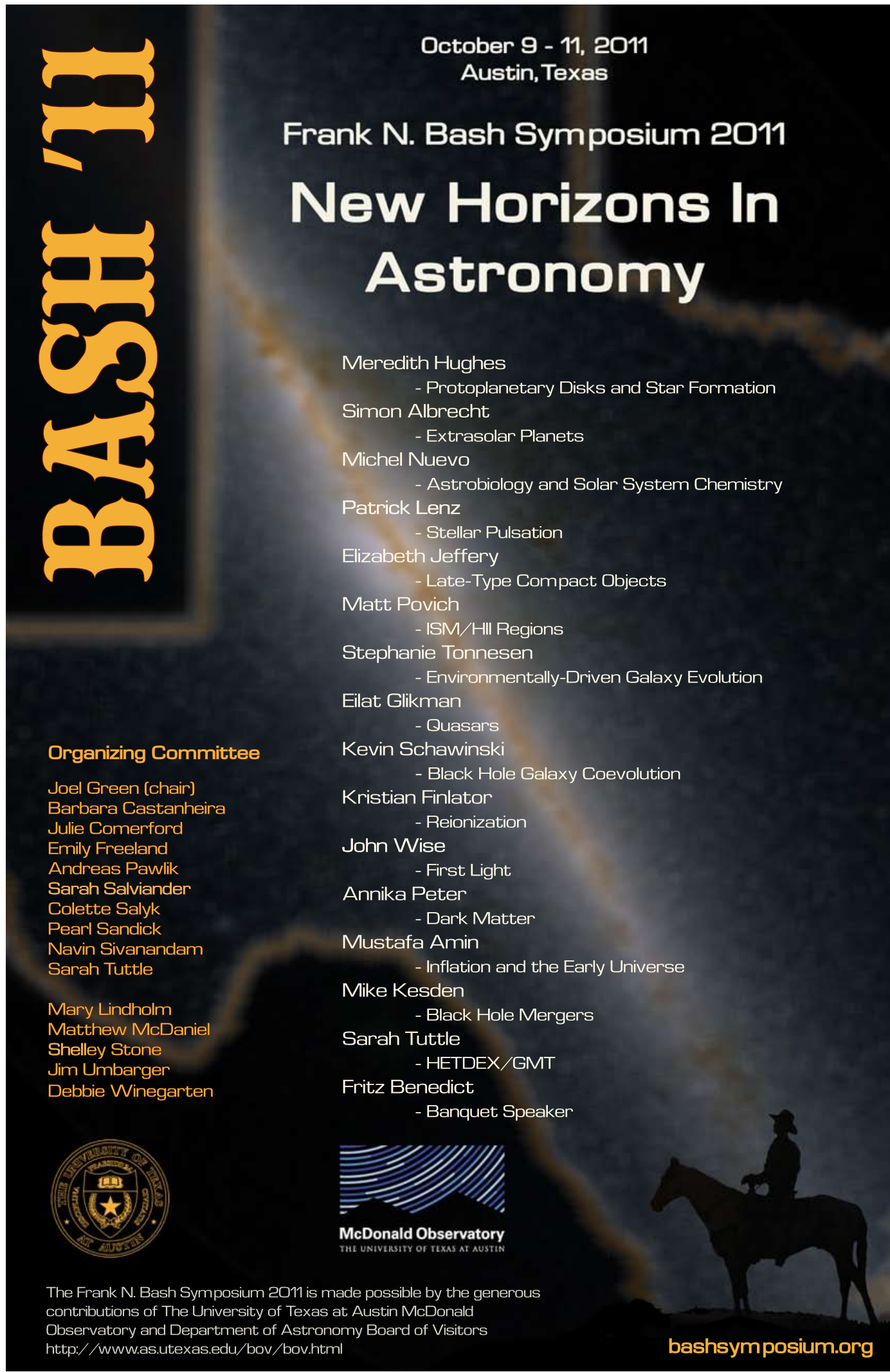




\section{Preface}

The 4th Frank N. Bash Symposium took place on October 9-11, 2011, in the central campus of the University of the Texas at Austin. The Symposium is held biannually in honor of former McDonald Observatory Director, Frank Bash. A total of fourteen review talks were presented by a first-class group of invited postdoctoral researchers. Their topics spanned all of modern astronomy, from the local universe to the very beginnings of space and time. Highlights were local researcher Sarah Tuttle's presentation of the UT role in the Giant Magellan Telescope project, as well as the posters by graduate students and undergraduates from universities around Texas. During the speaker and poster sessions, social luncheons, dinners, and meetings, a vigorous spirit of scientific inquiry and community was fostered between the research visitors, the faculty and staff of UT, and the McDonald Observatory Board of Visitors (which provides ongoing financial support for the Symposium). The Symposium was a great success, with 120 visitors from across Texas, the United States, and Europe. We demonstrated that it is truly possible to span the Universe in fifteen well-tuned talks when using engaging speakers selected from the ranks of enthusiastic young researchers.

An event like this requires the supporting efforts of many individuals. We would like to extend our thanks to the following people for their contributions to the Symposium: Associate Chair of Astronomy Karl Gebhardt for starting off the festivities, and Astronomy Department Chair Dan Jaffe for offering much-needed support and advice; all of the staff whose assistance made the Symposium run smoothly, including Shelley Stone, Matthew McDaniel, Mary Lindholm, Jim Umbarger, Kelly Quinney, Gordon Orris, and Debbie Winegarten, who collectively manned the registration desk, organized and scheduled events, managed poster stands, and crafted and updated the Bashfest webpage; Lara Eakins for providing a seamless audio-visual experience that kept the audience engaged throughout; Jim Umbarger and others who took photographs for the webpage and these proceedings; our banquet speaker, Fritz Benedict, for an entertaining and thought-provoking talk; Judit Györgyey Ries for her inventive poster design; Sarah Tuttle and Colette Salyk for their beautiful T-shirt design; postoc Mia Bovill for her enthusiastic and efficient organization of the flash poster talks; and past Bashfest organizers, particularly Remco van den Bosch and Tom Barnes, for guidance.

The sessions were chaired by Colette Salyk, Joel Green, Julie Comerford, and Andreas Pawlik. The SOC and LOC were combined for this occasion and included: Joel Green (chair), Barbara Castanheira, Julie Comerford, Emily Freeland (Texas A\&M University) Andreas Pawlik (proceedings editor), Sarah Salviander (proceedings senior editor), Colette Salyk, Pearl Sandick, Navin Sivanandam, and Sarah Tuttle (representing postdocs); additionally, Mary Lindholm, Matthew McDaniel, Shelley Stone, Jim Umbarger, Lara Eakins, Kelly Quinney, Gordon Orris, and Debbie Winegarten (representing staff). Finally, we would like to extend our heartfelt thanks to the McDonald Observatory Board of Visitors for their interest and support, both financial and moral, all of which makes this Symposium possible.

\section{On behalf of the LOC, SOC, and Proceedings Editors, Joel Green, Sarah Salviander \& Andreas Pawlik}




\section{Frank Bash}

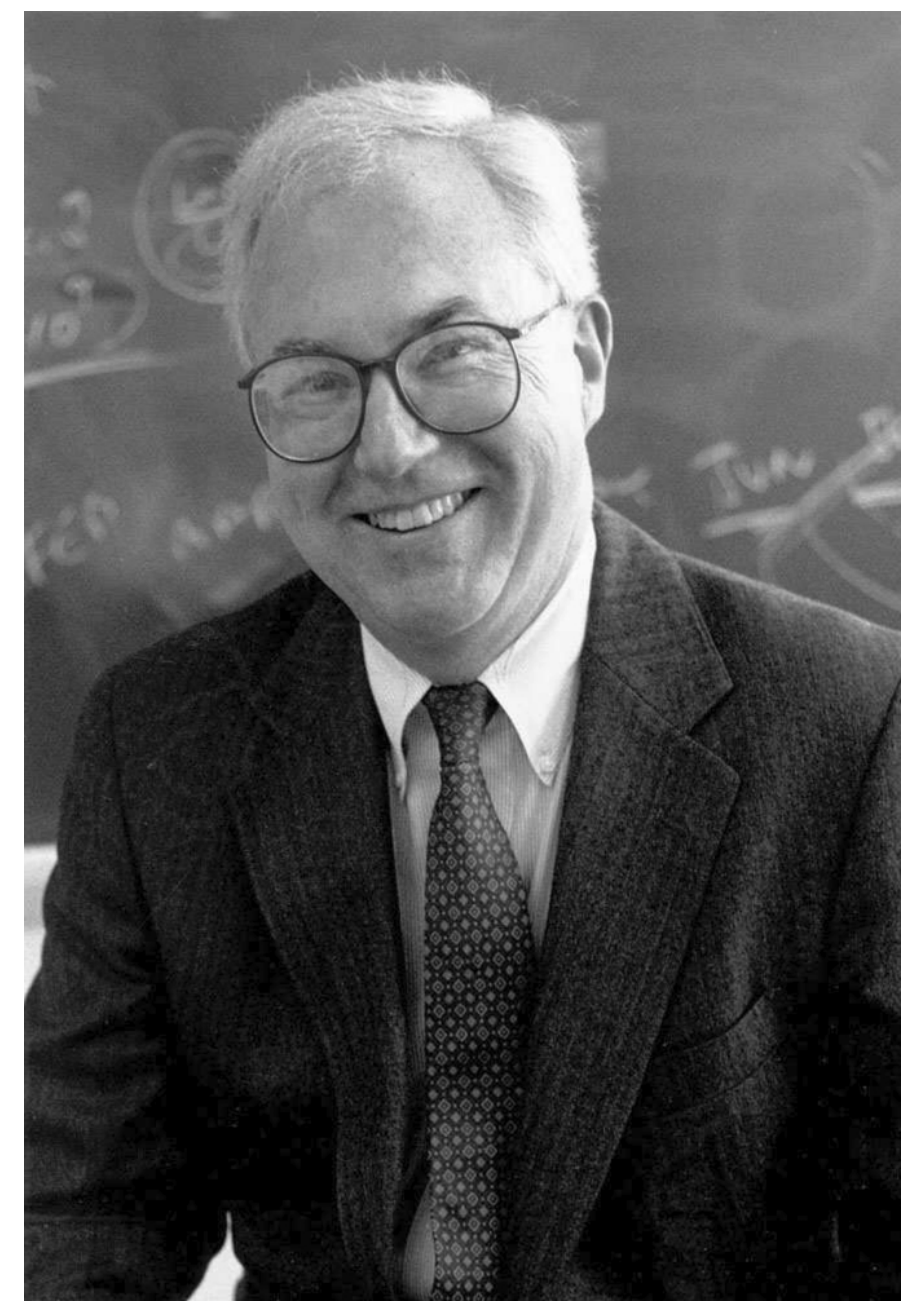

Frank N. Bash, Ph.D., served as director of McDonald Observatory from 1989-2003. A native of Medford, Oregon, Bash earned his bachelor's degree from Willamette University in Salem, Oregon; his master's degree in astronomy from Harvard University; and his doctorate from the University of Virginia. A well-known and widely published specialist in radio astronomy, Dr. Bash's research interests include large-scale formation processes in spiral galaxies. Dr. Bash joined the faculty of the University of Texas at Austin in 1969. Serving as Chairman of the Astronomy Department from 1982 through 1986, in 1985 he was named the Frank N. Edmonds Regents Professor. Among numerous awards for quality teaching, he was named to the teaching excellence Hall of Fame at UT Austin in 1984. As Director of McDonald, Dr. Bash led the effort for design, funding, and construction of the Hobby-Eberly Telescope. Dr. Bash also led the effort to expand the publicoutreach programs of the Observatory. These programs include the Observatory's Visitors Center, which hosts approximately 60,000 visitors per year, and StarDate radio, which reaches millions of people each day in English, Spanish, and German. 


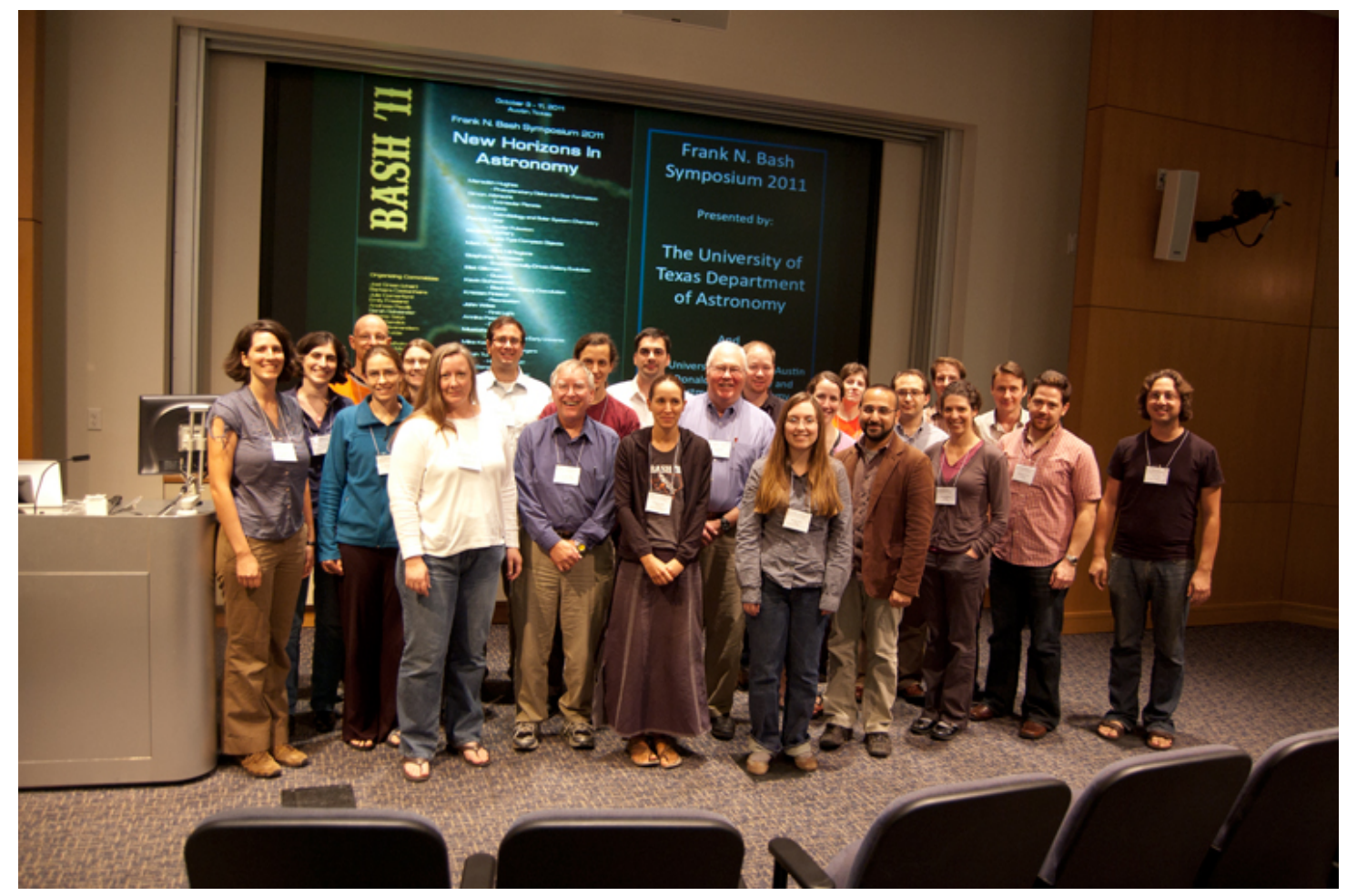

Frank N. Bash Symposium: New Horizons In Astronomy speakers and SOC 


\section{Speakers}

\section{Simon Albrecht}

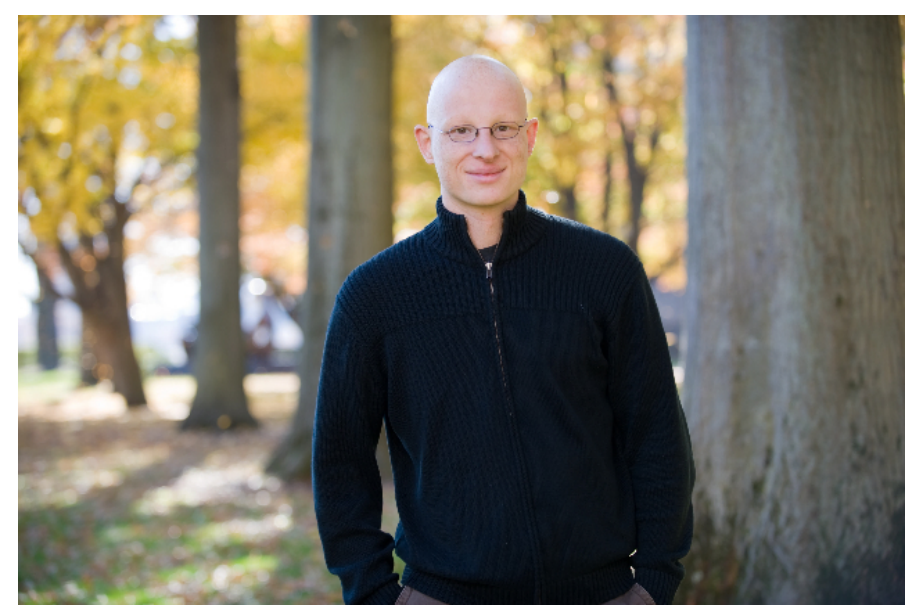

\section{Exoplanets}

In this paper I will discuss the current knowledge we have on planets orbiting other stars than our sun: exoplanets. Our ability to learn about exoplanets depends on the method we choose to search for them. Therefore I will give a short overview of the different detection techniques that are used to find planets. Special emphasis is placed on two techniques, radial velocity search and transit search.

I will also focus on a long standing riddle of the field: The occurrence of gas giant planets on orbits with periods of only a few days (Hot-Jupiters). These planets are similar to Jupiter but orbit their stars much closer than even Mercury orbits our own sun. The detection of these Hot-Jupiters was a surprise, as it is thought that they cannot form so close to their stars. Recent measurements of the Rossiter-McLaughlin effect helped to shed light on the formation and evolution of these systems.

Finally I will highlight some exciting new developments and recent detections of exoplanets in extreme environments. 


\section{Patrick Lenz}

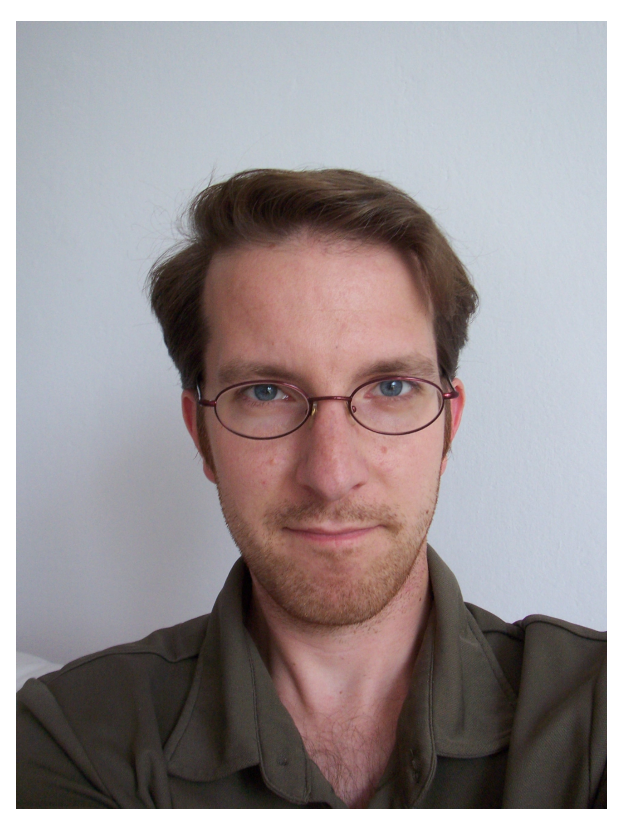

Asteroseismology of Stars on the Upper Main Sequence

I review the properties of pulsators located on the upper main sequence in the HR diagram and discuss asteroseismic inferences on the internal structure of stars of spectral type A and B. Special attention is given to the problem of uncertainties in stellar opacities in modeling. 


\section{Michel Nuevo}

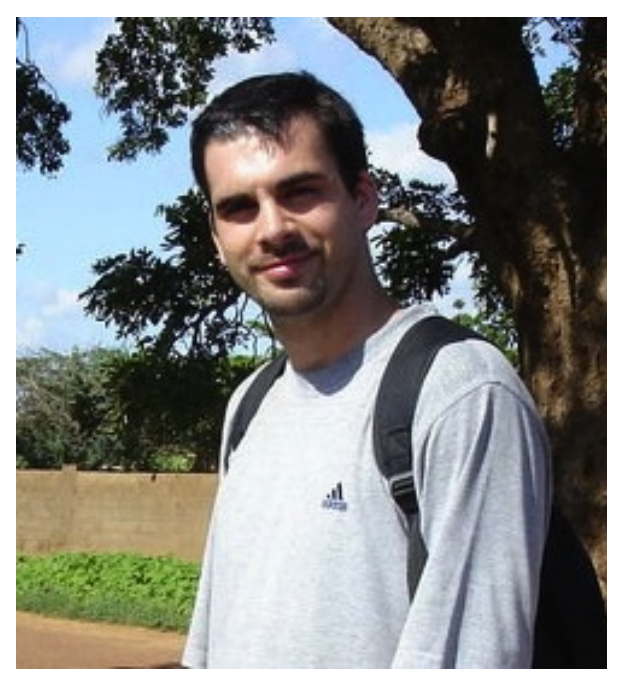

Laboratory Astrochemistry: A Powerful Tool to Understand the Origin of Organic Molecules in the Interstellar Medium, Comets, and Meteorites

During the past two decades, astrochemistry laboratory simulations have shown that complex organic molecules can be formed under simulated astrophysical conditions from the vacuum ultraviolet (UV) irradiation of ice mixtures containing simple species such as $\mathrm{H}_{2} \mathrm{O}, \mathrm{CO}, \mathrm{CO}_{2}, \mathrm{CH}_{3} \mathrm{OH}$, and $\mathrm{NH}_{3}$. These organics include compounds of biological and prebiotic interests such as amino acids - the building blocks of proteins, and nucleobases - the informational subunits of DNA and RNA. Although the presence of amino acids in the interstellar medium (ISM) has not been confirmed by observations to date, they have been detected in meteorites, indicating that biomolecules and/or their precursors can be formed under extraterrestrial, non-biological conditions. Nucleobases have also been detected in meteorites, broadening the variety of complex organic molecules that can be formed in astrophysical environments. Like amino acids, nucleobases and other $\mathrm{N}$ heterocycles have not been observed in the ISM. In the following, I will review some of the progress made by laboratory astrochemistry towards understanding the formation of organic species from the UV irradiation of ices at low temperature under astrophysically relevant conditions. This discussion will be focused on the formation of amino acids and other molecules of prebiotic interest such as urea and glycerol. Then, I will present recent studies on the for- mation of nucleobases and related compounds from the UV irradiation of pyrimidine in $\mathrm{H}_{2} \mathrm{O}, \mathrm{NH}_{3}$, and $\mathrm{CH}_{3} \mathrm{OH}$ ices, which show the formation of a large suite of photo-products including the nucleobases uracil and cytosine. 


\section{Elizabeth Jeffery}

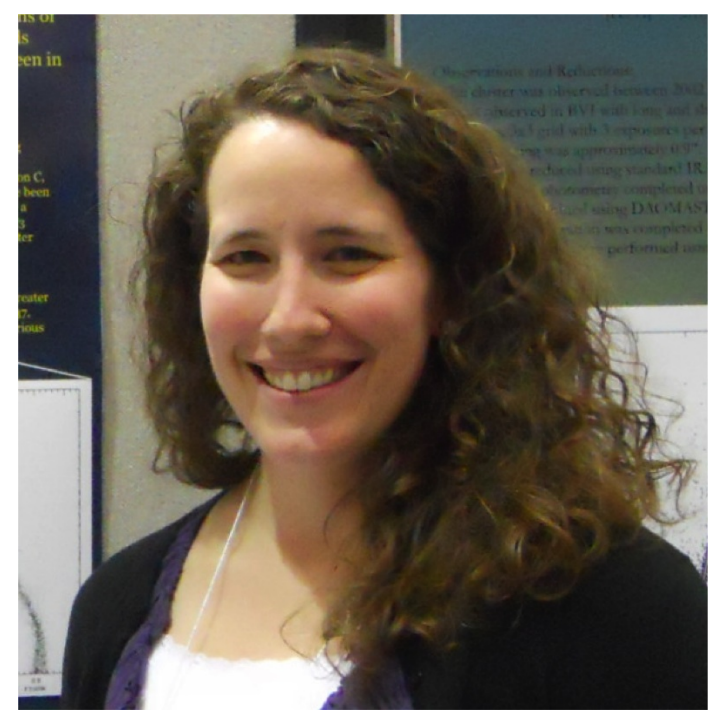

Late-type Compact Objects

The final stages of stellar evolution for a low mass star are fairly unspectacular when compared to their high mass siblings. Once the outer layers of a star are shed, the hot exposed core that's left behind and begins to cool. As it does so, it exhibits several interesting (and useful!) character traits. In this paper I will give an overview of the discovery of white dwarf stars and what we now understand about their formation and place within the story of stellar evolution. The fate of $\sim 98 \%$ of the stars in our Galaxy, white dwarfs continue to prove their usefulness and versatility as probes of understanding a number of interesting astrophysical problems. I will discuss a few of these applications, with particular emphasis on work done studying white dwarfs in star clusters. 


\section{Matt Povich}

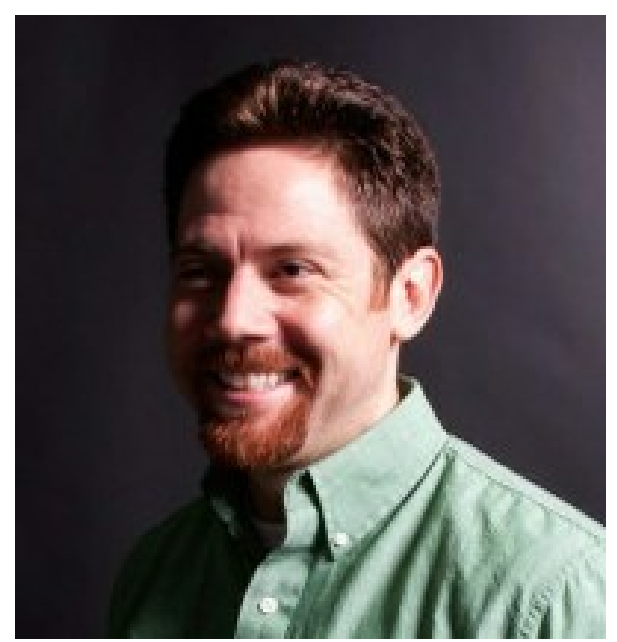

Beyond Strömgren Spheres and Wind-Blown Bubbles: An Observational Perspective on $\mathrm{H}$ II Region Feedback

Massive stars produce copious quantities of ultraviolet radiation beyond the Lyman limit, photoionizing the interstellar medium (ISM) and producing H II regions. As strong sources of recombination- and forbidden-line emission, infrared continuum, and thermal (free-free) radio continuum, H II regions serve as readily-observable beacons of massive star formation in the Milky Way and external galaxies. Along with supernovae, H II regions are dominant sources of feedback in star-forming galaxies, injecting radiative and mechanical luminosity into the ISM. H II regions may prove more important than supernovae as triggers of star formation through localized compression of cold cloud cores. In this review, I give a broad overview of the structure and time-evolution of $\mathrm{H}$ II regions, emphasizing complications to the theoretical picture revealed by multiwavelength observations. I discuss a recent controversy surrounding the dominant feed- back mechanism in 30 Doradus, the most luminous H II region in the Local Group. I summarize the first results from the Milky Way Project (MWP), which has produced a new catalog of several thousand candidate Galactic H II regions by enlisting > 35, 000 "citizen scientists" to search Spitzer Space Telescope survey images for bubble-shaped structures. The MWP and similar large catalogs enable empirical studies of Galactic H II region evolution across the full range of luminosities and statistical studies of triggered star formation. 


\section{Michael Kesden}

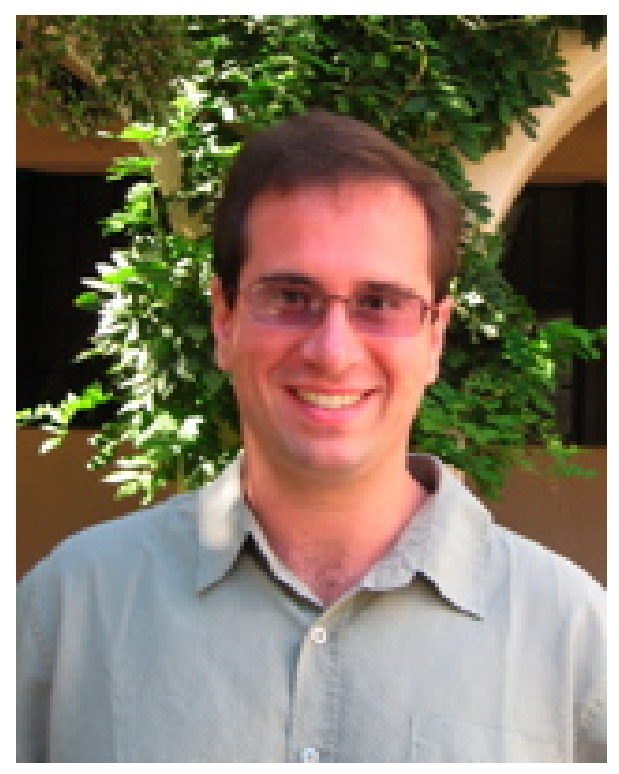

Black-Hole Mergers

Observations of black-hole mergers will provide unique insights into both general relativity and cosmology. The gravitational waves produced in such mergers directly probe strong-field solutions to Einstein's equations, and the rate at which mergers occur constrains the hierarchical formation of supermassive black holes and their host galaxies. The three primary theoretical tools for studying merging black holes are post-Newtonian expansions, black-hole perturbation theory, and numerical relativity. I will briefly explain how these tools complement each other in developing a general solution to the problem of black-hole mergers, highlighting some of my own research in each of these three areas. 


\section{Stephanie Tonnesen}

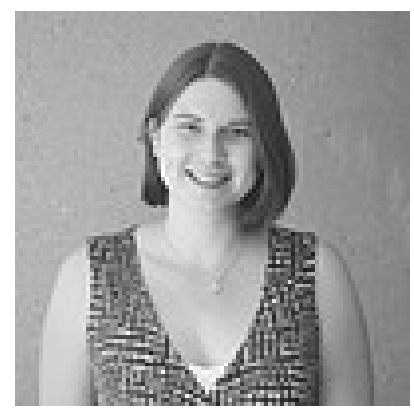

\section{Environmentally-Driven Galaxy Evolution}

The galaxy population in clusters differs from that in the field. The cluster galaxy population (at $z \leq 0.4$ ) is dominated by red, early-type galaxies. Although some of this can be explained by the mass-morphology relation, in this proceedings I discuss work that has shown that, particularly in low-mass galaxies, there is environmentally-driven evolution from blue (star-forming) to red (non-star-forming). While environment can be measured in several ways, I show that local environment affects galaxies up to the scale of the halo in which they reside. Focusing on clusters, I introduce the different mechanisms that can affect galaxies: galaxy-cluster, galaxy-galaxy, and galaxy-intracluster medium interactions. I then give an overview of the evidence that the colorevolution of galaxies from blue to red is reflected in the morphological evolution of galaxies from spiral to S0, and discuss the necessary steps in the evolution of spirals into S0s. I then briefly describe some of my own research examining the role of ram pressure stripping on the morphological evolution of spiral galaxies. While ram pressure stripping can effectively remove the fuel for star formation and redden galaxies, this will form S0s that are less luminous than their spiral progenitors. Therefore, while ram pressure stripping may be an important mechanism driving the formation of S0s from spirals, it cannot be the only process driving this morphological evolution. 


\section{Eilat Glikman}

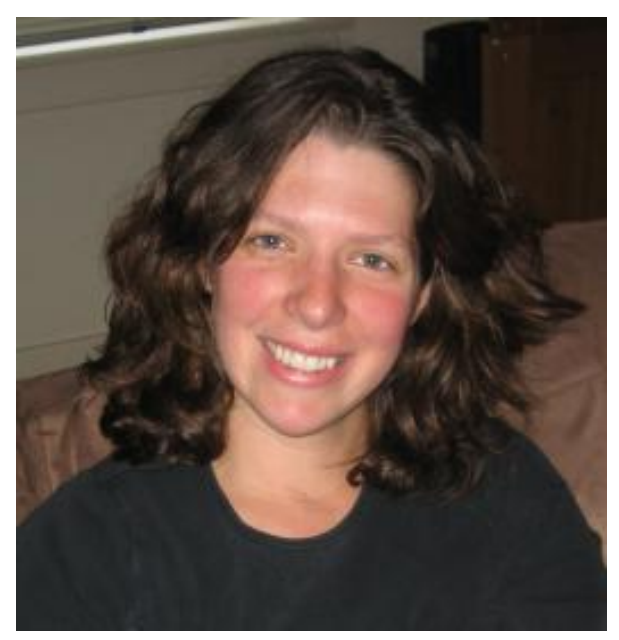

The Reddest Quasars: A Transitional Phase in Quasar/Galaxy Co-Evolution

Quasars are extremely luminous sources powered by accretion of gas onto a supermassive black hole in the nucleus of some galaxies. Most of the $>10^{5}$ quasars identified in the literature have been identified in optical surveys through the "ultraviolet excess" (UVX) method. However, these samples are known to be incomplete and biased because of obscuration and anisotropic radiation. To overcome some of these biases and search for candidate obscured quasars, we matched radio sources from the FIRST $1.4 \mathrm{GHz}$ survey with the 2MASS near-infrared survey and selected objects with red optical-to-near-infrared colors. We followed up our candidates with optical and/or near-infrared spectroscopy and identified 119 dust-reddened quasars, defined as having at least one broad emission line in and a reddening of $E(B-V)>0.1$. The sample spans a wide redshift range, $0.1<z<3$ and reaches a reddening, $E(B-V) \lesssim 1.5$. When corrected for extinction, red quasars are the most luminous objects at every redshift and the fraction of red quasars increases with luminosity. The properties of red quasars suggest that they are revealing an evolutionary phase where the heavily obscured quasar is emerging from its dusty environment prior to becoming a "normal" blue quasar. We compute the fraction of quasars that are in this red phase and determine that its duration is $\sim 20 \%$ as long as the unobscured quasars phase: a few million years. 


\section{Kevin Schawinski}

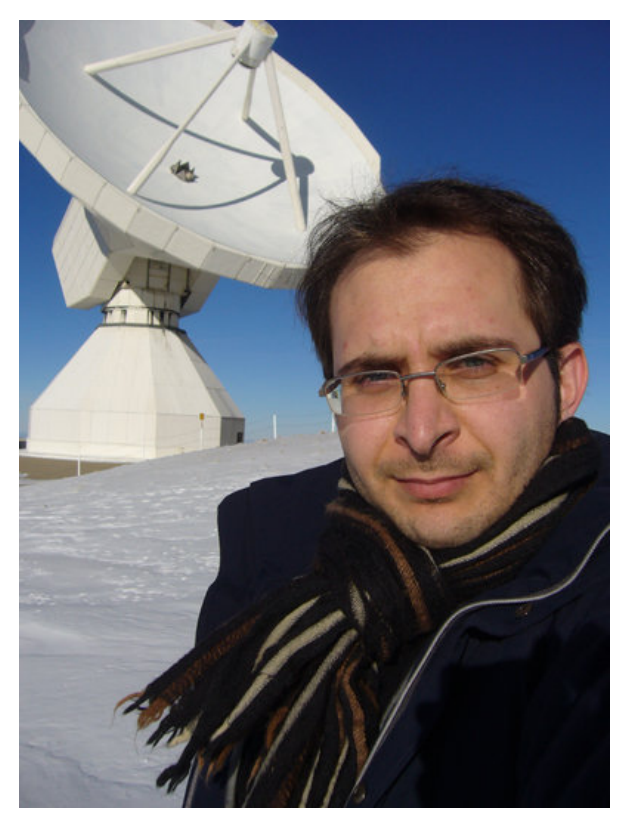

\section{Black Hole Galaxy Coevolution}

The growth of black holes and the formation and evolution of galaxies appear to be linked at such a fundamental level that we think of the two as 'co-evolving.' Recent observations show that this co-evolution may be complex and be the result of several different pathways. While it is clear that black hole accretion is linked to specific phases of the evolution of the host galaxy, the impact of the energy liberated by the black hole on the evolutionary trajectory of the host by feedback is less clear. In this contribution, I review the motivations for co-evolution, the current state of the observational picture, and some challenges by black hole feedback. 


\section{Sarah Tuttle}

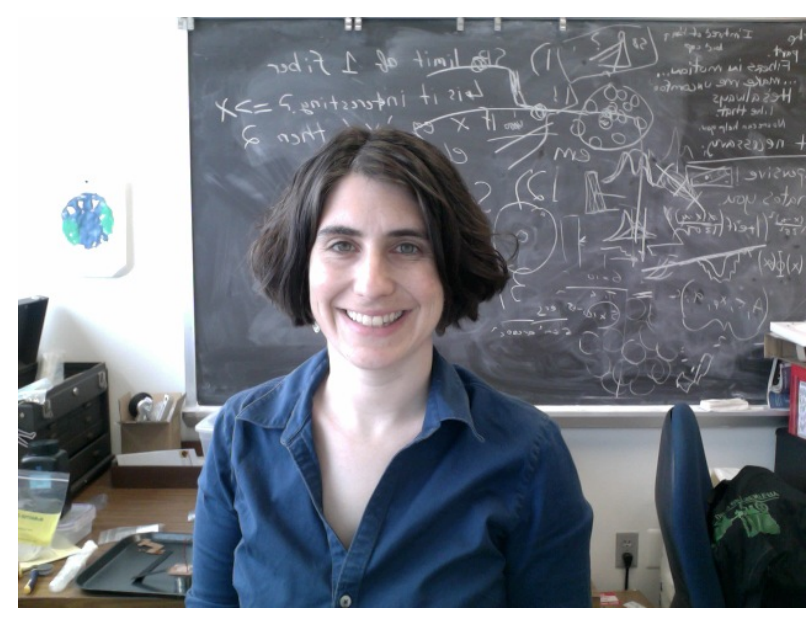

Spectroscopy and the Age of Giant Telescopes

Is your 4 meter telescope just not cutting it anymore? Embarrassed to mention the telescope time you had just the other week when talking about your newest data project? Don't worry, the age of the giant telescope is upon us. I will review the status of the three current ELT projects, as well as spectroscopic technologies focusing on multi-object spectroscopy. HETDEX (the Hobby Eberly Dark Energy Experiment) uses a new approach to large instruments, replicating a spectrograph channel 150 times to produce 33,600 individual spectra across a 22 arcminute field. The technology being built can be easily modified and ported to other telescopes to take quick advantage of the integral field approach. I will touch on the many experiments that await first light as these instruments come to fruition. Let's discuss why we are pouring massive resources into these telescopes and see what they can do for us. 


\section{Kristian Finlator}

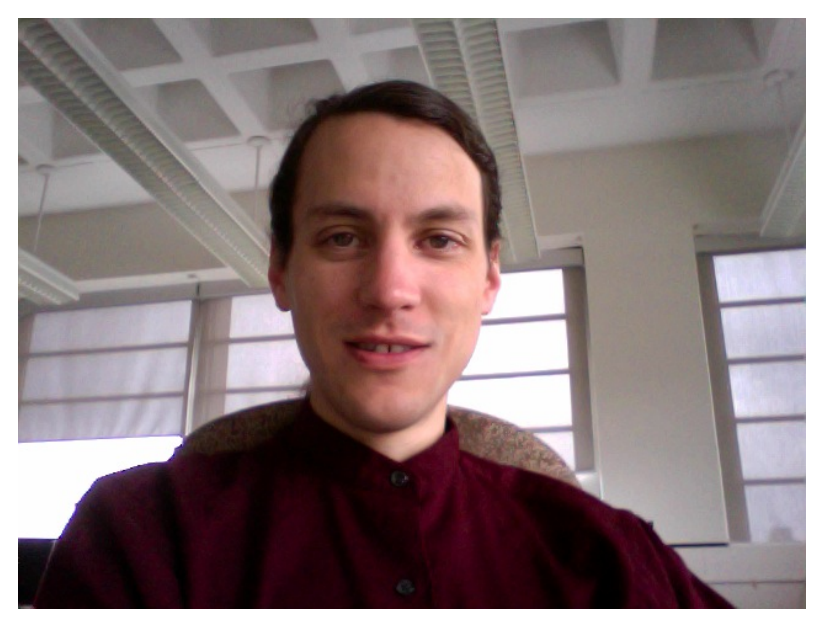

Recent Advances in Cosmological Hydrogen Reionization

I discuss recent advances in the study of hydrogen reionization, focusing on progress that was achieved during the years 2010-2011. First, I discuss recent measurements of the progress of reionization. Next, I discuss recent observational constraints on the nature and abundance of the dominant ionizing sources. Finally, I discuss recent progress in modeling reionization. This review is written for an audience of astronomers who do not specialize in the high-redshift Universe. 


\section{John Wise}

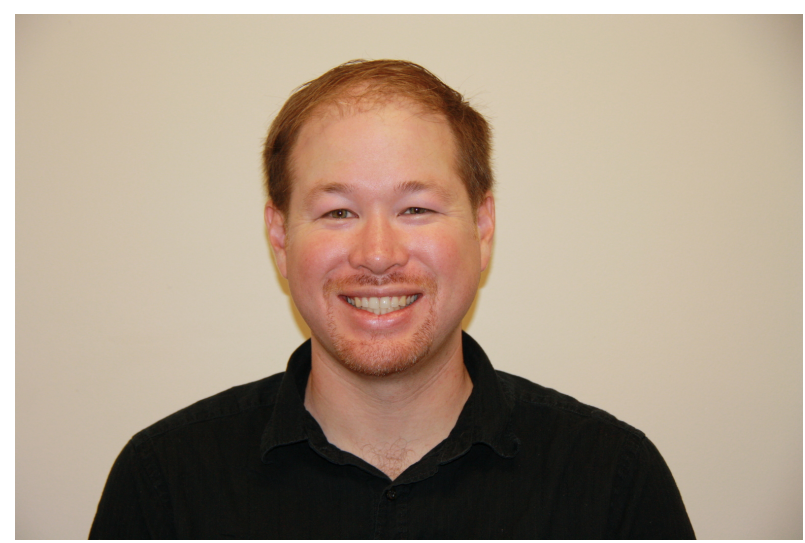

First Light

The first stars in the universe are thought to be massive, forming in dark matter halos with masses around $10^{6}$ solar masses. Recent simulations suggest that these metal-free (Population III) stars may form in binary or multiple systems. Because of their high stellar masses and small host halos, their feedback ionizes the surrounding $3 \mathrm{kpc}$ of intergalactic medium and drives the majority of the gas from the potential well. The next generation of stars then must form in this gas-poor environment, creating the first galaxies that produce the majority of ionizing radiation during cosmic reionization. I will review the latest developments in the field of Population III star formation and feedback and its impact on galaxy formation prior to reionization. In particular, I will focus on the numerical simulations that have demonstrated this sequence of events, ultimately leading to cosmic reionization. 


\section{Annika Peter}

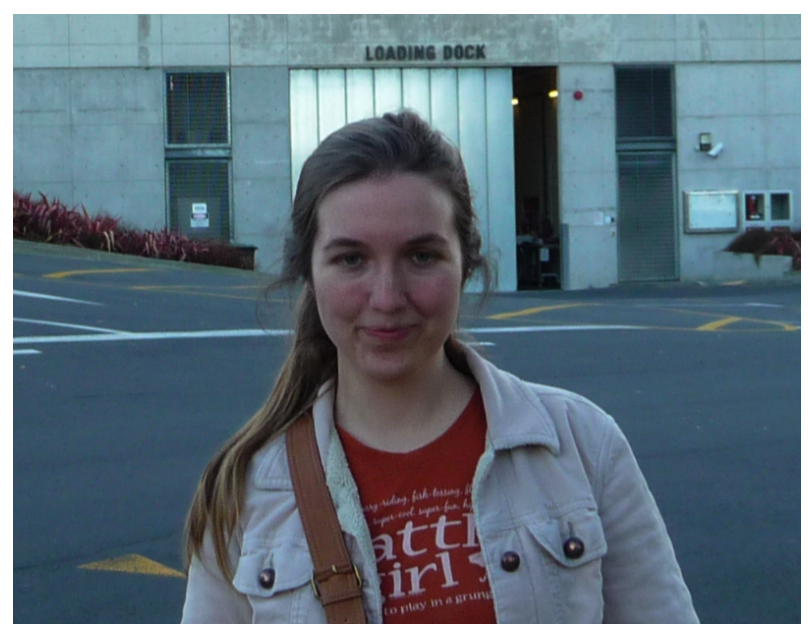

\section{Dark Matter}

From astronomical observations, we know that dark matter exists, makes up $23 \%$ of the mass budget of the Universe, clusters strongly to form the load-bearing frame of structure for galaxy formation, and hardly interacts with ordinary matter except gravitationally. However, this information is not enough to identify the particle specie(s) that make up dark matter. As such, the problem of determining the identity of dark matter has largely shifted to the fields of astroparticle and particle physics. In this talk, I will review the current status of the search for the nature of dark matter. I will provide an introduction to possible particle candidates for dark matter and highlight recent experimental astroparticle- and particle-physics results that constrain the properties of those candidates. Given the absence of detections in those experiments, I will advocate a return of the problem of dark-matter identification to astronomy, and show what kinds of theoretical and observational work might be used to pin down the nature of dark matter once and for all. This talk is intended for a broad astronomy audience. 


\section{Poster Presenters}

\section{Taylor S. Chonis}

Photometry of the Stellar Tidal Stream in the Halo of Messier 63

\section{Anson D'Aloisio}

The Effects of Primordial Non-Gaussianity on Giant-Arc Statistics: A Scale-Dependent Example

\section{Amanda Heiderman}

The VIRUS-P Investigation of the eXtreme Environments of Starbursts (VIXENS): Survey and First Results

\section{Jacob A. Hummel}

The Hunt for the First Supernovae: The Source Density and Observability of Pair-Instability Supernovae from the First Stars

\section{John R. Jardel}

Integral Field Spectroscopy of the Center of the Draco Dwarf Spheroidal

\section{Myoungwon Jeon}

The First Galaxies: Assembly with Black Hole Feedback

\section{Hyo Jeong Kim}

Evidence of Episodic Accretion in Spitzer IRS Spectra of Low-Luminosity Embedded Protostars

\section{Hyunbae Park}

Revisiting the Post-reionization Kinetic Sunyaev-Zel'dovich Effect on the Cosmic Microwave Background Fluctuations

\section{Chalence Safranek-Shrader}

Primordial Star Formation in the First Galaxies 